\title{
Location-Dependent Prediction of Dynamic Stability Limit for Peripheral Milling of Surfaces with Variable Curvatures
}

\author{
Baosheng Wang $\mathbb{D}^{1},{ }^{1}$ Hongyan Hao, ${ }^{1}$ Mulan Wang, ${ }^{2}$ and Junming Hou ${ }^{2}$ \\ ${ }^{1}$ Research Department of Intelligent Manufacturing Equipment, Nanjing Institute of Technology, Nanjing 211167, China \\ ${ }^{2}$ Jiangsu Key Laboratory of Advanced Numerical Control Technology, Nanjing 211167, China \\ Correspondence should be addressed to Baosheng Wang; wbaosh@163.com
}

Received 13 September 2017; Revised 15 January 2018; Accepted 21 February 2018; Published 3 May 2018

Academic Editor: Mickaël Lallart

Copyright (c) 2018 Baosheng Wang et al. This is an open access article distributed under the Creative Commons Attribution License, which permits unrestricted use, distribution, and reproduction in any medium, provided the original work is properly cited.

\begin{abstract}
The stability limit may change with the cutter's location due to effect of curvature during the milling of a complex surface. The method for calculating the actual radial cutting depth is presented by accounting for the effects of curvature on the actual cutting parameters. The computed radial cutting depth is in turn used to determine the entrance/exit angles. Moreover, a milling system dynamic model is established based on the instantaneous milling force coefficients, and the stability limit is determined by means of the time-domain semidiscretization method. In addition, a location-dependent method for predicting the stability associated with the peripheral milling of a complex surface is put forward and simulation is carried out to generate a stability limit diagram. The effectiveness of the proposed method is verified through milling tests.
\end{abstract}

\section{Introduction}

Cutting vibration may influence the quality and efficiency of the machining process or even lead to damage of cutters or milling machines. For this reason, it is desired to suppress the cutting vibration to the extent possible during the machining process. It is a traditional practice to select conservative processing parameters for preventing the cutting vibration, whose disadvantages include reduced production efficiency and machine utilization. Therefore, characterizing the milling system's cutting vibration pattern and determining the range of operating conditions for ensuring the stable milling operation have become an important research area concerning the high speed milling.

Currently, scholars at home and abroad put forward many methods for predicting the chatter stability of a milling system. Altintaş and Budak first propose a single-frequency method $[1,2]$, which utilizes the zero-order term of the Fourier series associated with the coefficient matrix in the milling force direction to linearize the mathematical model so as to allow analytical calculation of the stability boundary. Merdol put forward a multifrequency method, which collectively takes into consideration a multitude of harmonics associated with the Fourier series of the coefficient matrix in the milling force direction [3]. Insperger presents a semidiscretization method in time domain, which, by discretizing the delay term, converts the delay differential equations governing the milling dynamics into a set of ordinary differential equations; this allows the construction of a system state transition matrix, based on which the stability of the system can be assessed using Floquet's theory [4]; moreover, Zatarain employs this method to analyze the impact of milling cutter's helix angle on the stability boundary by means of this method [5], and Long examines the effect of speed variation on the chatter stability [6]. Guo studies the milling stability limit associated with the multiregenerative effects based on the 3rd-order semidiscretization method [7]. Ding proposes a full-discretization method, which, by fully discretizing the delay and state terms, allows the application of Floquet's theory to determine the stability limit of the system [8]. Ozoegwu carries out analysis concerning the impact of the discretization order on the prediction accuracy for the fulldiscretization method [9]. Zatarain proposes an implicit subspace iteration approach to enhance the efficiency of calculating the stability limit diagram, which is compatible with both semidiscretization and full-discretization methods 
in time [10]. Constant milling force coefficients are utilized for calculating the dynamic milling force in [1-10]; hence, the effect of feed per tooth on the chatter stability is neglected. Landers establishes a dynamic model based on the instantaneous milling force coefficients in exponential form so as to analyze the effect of feed per tooth on the chatter stability [11]; moreover, Ahmadi studies the linear milling stability with varying radial depth of cut based on the instantaneous milling force coefficients in exponential form [12].

It should be noted that the previous studies cited above [1-12] are all focused on linear milling. Kardes studies the stability limit of the circular milling process using singlefrequency method [13], and Peng analyzes the chatter stability during a circular corner milling process based on the timedomain simulation method [14]. Unlike the linear and circular milling, during the peripheral milling of a surface with variable curvature, tool feed direction and the actual radial cutting depth are changing continuously along tool path. Then, milling dynamics and stability characteristics are also changing along tool path. Yang presented a method to predict chatter for the peripheral milling of thin-walled workpieces with curved surfaces [15]. In the paper, the milling force coefficients are simplified as constants. So, the feed per tooth cannot be included in dynamic model, and limit of feed per tooth cannot be predicted.

In the present study, effective radial cut depth is proposed, and a new milling force model is established. With the milling force model, a dynamic model of cutting tools is constructed. Subsequently, the time-domain semidiscretization method is employed to calculate the stability limit. Considering that the actual radial cutting depth and tool feed direction are changing along tool path, a new method for location-dependent prediction of dynamic stability limit for peripheral milling of surfaces with variable curvatures is presented. Moreover, the proposed algorithm is experimentally validated. The advantage of the proposed approach lies in that stability during milling process can be predicted. Thus, stable milling will be ensured.

\section{Dynamic Model for Peripheral Milling of Complex Surface}

2.1. Geometric Description of Peripheral Milling of Complex Surface. The dynamic model of a milling system for peripheral milling of a complex surface is schematically shown in Figure 1, where $\left(x_{t}(u), y_{t}(u)\right),\left(x_{w}(u), y_{w}(u)\right)$, and $(x(u), y(u))$ represent the parameterized tool path, contour prior to processing, and target contour, respectively. oxy and $o_{s} x_{s} y_{s}$ denote the fixed and follow-up coordinate systems, respectively. $R$ represents the tool radius. $a_{e}$ represents the nominal radial depth of cut. $\theta(t)$ represents the instantaneous feed angle.

Regarding the parametric geometry of the target contour for reference, the tool path and contour prior to processing are expressed as

$$
\begin{aligned}
& x_{w}(u)=x(u)-\frac{a_{e} y^{\prime}(u)}{\sqrt{x^{\prime}(u)^{2}+y^{\prime}(u)^{2}}} \\
& y_{w}(u)=y(u)+\frac{a_{e} x^{\prime}(u)}{\sqrt{x^{\prime}(u)^{2}+y^{\prime}(u)^{2}}}
\end{aligned}
$$

$$
\begin{aligned}
& x_{t}(u)=x(u)-\frac{R y^{\prime}(u)}{\sqrt{x^{\prime}(u)^{2}+y^{\prime}(u)^{2}}} \\
& y_{t}(u)=y(u)+\frac{R x^{\prime}(u)}{\sqrt{x^{\prime}(u)^{2}+y^{\prime}(u)^{2}}},
\end{aligned}
$$

where $x^{\prime}(u)$ and $y^{\prime}(u)$ represent the derivatives of the parametric curve, respectively.

During a machining process, the congruent relationship between the machining time $(t)$ and the parameter $(u)$ is calculated by

$$
t=\frac{60}{f_{z} N_{f} n_{r}} \sum_{i=1}^{u / \Delta u} L((i-1) \Delta u, i \Delta u)
$$

where $n_{r}$ represents the spindle speed. $f_{z}$ represents the nominal feed per tooth. $N_{f}$ represents the number of cutter teeth. $\Delta u$ represents the parameter spacing. $L((k-1) \Delta u, k \Delta u)$ represents the spacing between two adjacent parameter points on a tool path.

2.2. Dynamic Model. Our model, as schematically shown in Figure 2, consists of a rigid workpiece and a tool vibration system with two structural degrees of freedom. By accounting for only a single vibration mode in lieu of the two coupled vibration modes associated with the motions in two directions, the tool vibration can be described by the following differential equation:

$$
\mathbf{M} \ddot{\mathbf{p}}+\mathbf{C} \dot{\mathbf{p}}+\mathbf{K} \mathbf{p}=\mathbf{F},
$$

where $\mathbf{M}=\left[\begin{array}{cc}m_{x} & 0 \\ 0 & m_{y}\end{array}\right] ; \mathbf{C}=\left[\begin{array}{cc}c_{x} & 0 \\ 0 & c_{y}\end{array}\right] ; \mathbf{K}=\left[\begin{array}{cc}k_{x} & 0 \\ 0 & k_{y}\end{array}\right] ; \mathbf{P}=\left[\begin{array}{c}x(t) \\ y(t)\end{array}\right]$; and $\mathbf{F}=\left[\begin{array}{l}F_{x}(t) \\ F_{y}(t)\end{array}\right] \cdot m_{x}, c_{x}$, and $k_{x}$ represent the modal mass, damping coefficient, and stiffness in the $x$ direction, respectively. Similarly, $m_{y}, c_{y}$, and $k_{y}$ represent the modal mass, damping coefficient, and stiffness in the $y$ direction, respectively. $x(t)$ and $y(t)$ represent the instantaneous position at time $(t)$ in the fixed coordinate system $(x o y) . F_{x}(t)$ and $F_{y}(t)$ represent the instantaneous milling forces in the $x$ and $y$ directions, respectively.

By taking the tool's helix angle into account and dividing the milling cutter equally into an array of infinitesimal elements along the axial direction, the milling force associated with the element $(i, j)$ at time $t$ can be expressed as

$$
\begin{aligned}
d F_{t i, j}(t) & =K_{t}\left(h_{i, j}{ }^{\text {total }}(t)\right) h_{i, j}{ }^{\text {total }}(t) d z \\
d F_{r i, j}(t) & =K_{r}\left(h_{i, j}{ }^{\text {total }}(t)\right) h_{i, j}{ }^{\text {total }}(t) d z \\
& \left(i=1,2, \ldots, N_{f} ; j=1,2, \ldots, M\right),
\end{aligned}
$$

where $d F_{t i, j}(t)$ and $d F_{r i, j}(t)$ represent the tangential and radial milling forces, respectively. $h_{i, j}{ }^{\text {total }}(t)$ represents the instantaneous undeformed chip thickness. $K_{t}\left(h_{i, j}{ }^{\text {total }}(t)\right)$ and 


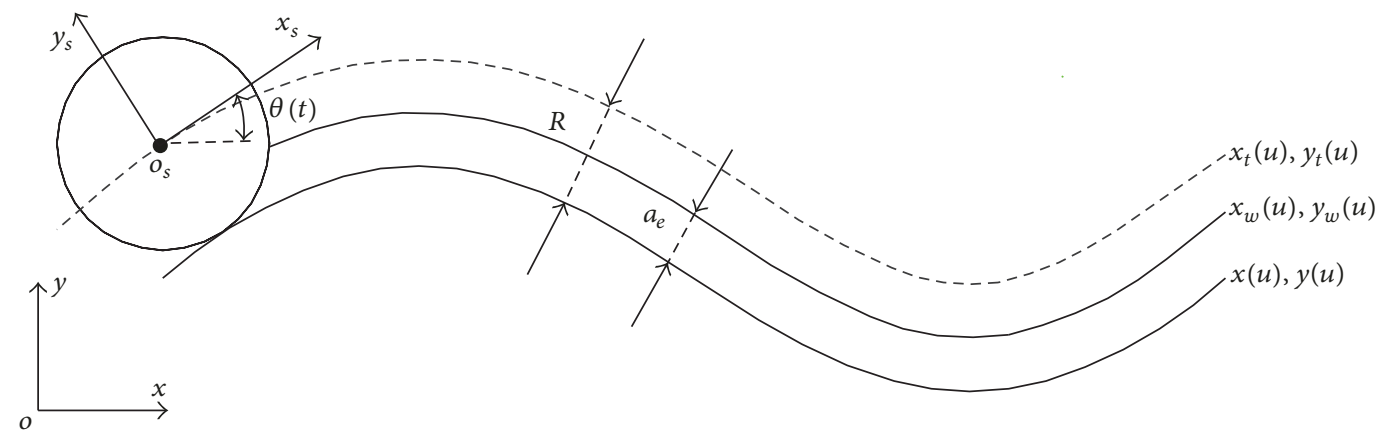

FIGURE 1: Schematic diagram of the proposed dynamic model describing the peripheral milling system for complex surface machining.

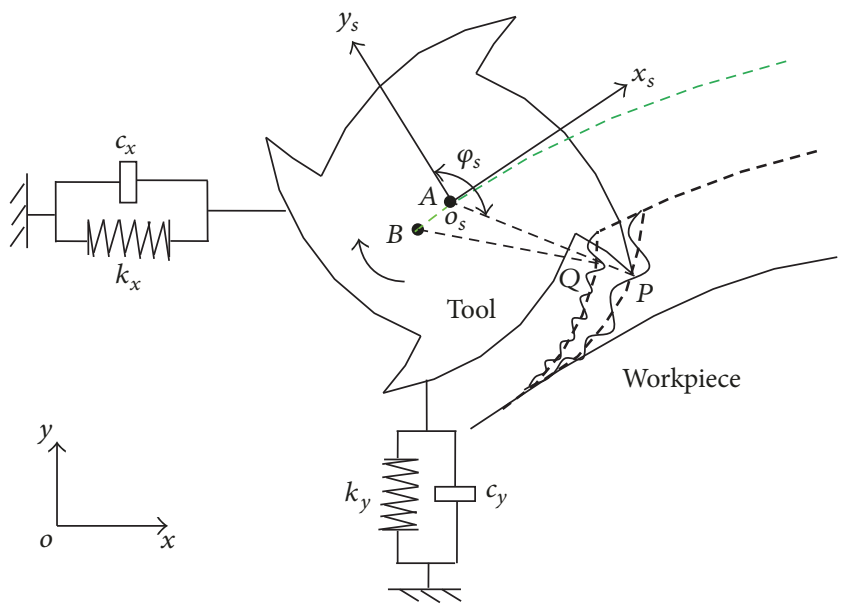

FIGURE 2: Schematic diagram of the proposed dynamic model describing a peripheral milling system.

$K_{r}\left(h_{i, j}{ }^{\text {total }}(t)\right)$ represent the tangential and radial milling force coefficients, respectively. $d z$ represents the axial length, and $M$ represents the number of elements per tooth.

The tool vibration typically leads to complicated chatter marks on the surface of the workpiece, and therefore the undeformed chip thickness for the next tooth may be influenced. For this reason, the instantaneous undeformed chip thickness needs to include two components, namely, the static undeformed chip thickness and the regenerative thickness due to cutting tool vibration, which can be calculated by the following equations:

$$
\begin{aligned}
h_{i, j}{ }^{\text {total }}(t) & =g\left(\varphi_{s i, j}(t)\right)\left({h_{i, j}}^{N}(t)+{h_{i, j}}^{S}(t)\right) \\
\varphi_{s i, j}(t) & =\varphi_{s}(t)-\frac{2 \pi(i-1)}{N_{f}}-\frac{(j-0.5) \tan \beta d z}{R} \\
\varphi_{s}(t) & =2 \pi n_{r} t+\theta(t),
\end{aligned}
$$

where $h_{i, j}{ }^{N}(t)$ and $h_{i, j}{ }^{S}(t)$ represent the static undeformed chip thickness and the regenerative undeformed chip thickness, respectively; $g\left(\varphi_{s i, j}(t)\right)$ represents the Heaviside step function; $\varphi_{s i, j}(t)$ represents the angular position at time $t$ in the follow-up coordinate system $\left(o_{s} x_{s} y_{s}\right) ; \beta$ represents the tool's helix angle; and $\varphi_{s}(t)$ represents the angular position at time $t$ in the follow-up coordinate system $\left(o_{s} x_{s} y_{s}\right)$.

It is found in our previous studies that the effect of the curvature on the actual feed per tooth is negligibly small during the peripheral milling process on a surface with variable curvature, and therefore the time-dependent feed per tooth can be approximated using a constant nominal feed per tooth [16]. Thus, the static undeformed chip thickness may be expressed as

$$
h_{i, j}{ }^{N}(t)=f_{z} \sin \left(\varphi_{s i, j}(t)\right) .
$$

Figure 2 indicates that the regenerative thickness $h_{i, j}{ }^{S}(t)$ corresponds to the difference between the tool's dynamic displacements at time $t$ (Point $A$ ) and time $t-T$ (Point $B$ ), which may be described as

$$
\begin{aligned}
h_{i, j}^{S}(t)= & {[x(t)-x(t-T)] \sin \left(\varphi_{i, j}(t)\right) } \\
& +[y(t)-y(t-T)] \cos \left(\varphi_{i, j}(t)\right),
\end{aligned}
$$

where $T$ represents the regenerative delay, and one can get $T=60 / N_{f} n_{r}$ by neglecting the effect of the surface curvature on the delay. $\varphi_{i, j}(t)$ represents the angular position at time $t$ in the fixed coordinate system $(x o y)$, and $\varphi_{i, j}(t)=\varphi_{s i, j}(t)-\theta(t)$.

During the peripheral milling process on a surface with variable curvature, the variation of the actual radial depth of cut causes the entrance/exit angles of the cutter teeth to change accordingly. Whether an element is involved in milling can be determined by the following equations:

$$
\begin{aligned}
& g\left(\varphi_{s i, j}(t)\right)= \begin{cases}1 & \phi_{\mathrm{st}}(t) \leq \varphi_{s i, j}(t) \leq \phi_{\mathrm{ex}}(t) \\
0 & \text { else }\end{cases} \\
& \phi_{\mathrm{st}}(t)=\pi-\arccos \left(1-\frac{a_{e e}(u)}{R}\right), \\
& \phi_{\mathrm{ex}}(t)=\pi
\end{aligned}
$$




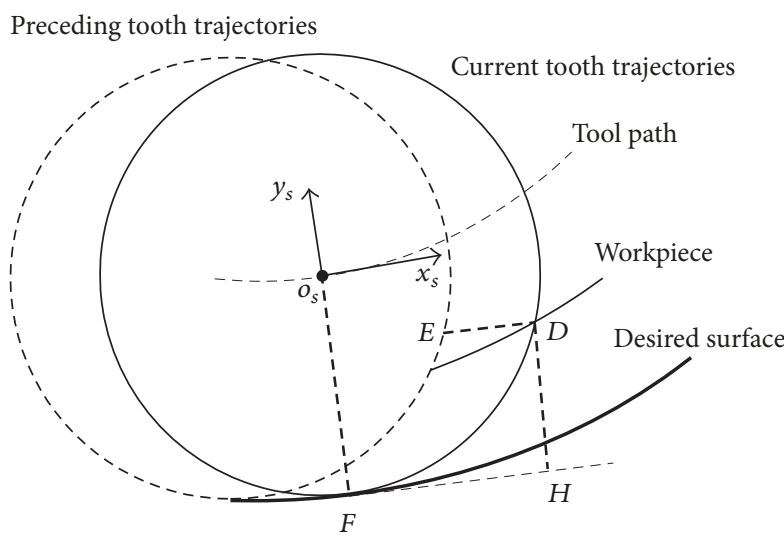

FIgUre 3: Actual radial cutting depth.

$$
\begin{aligned}
& \phi_{\text {st }}(t)=0, \\
& \phi_{\text {ex }}(t)=\arccos \left(1-\frac{a_{e e}(u)}{R}\right)
\end{aligned}
$$

up milling

$$
\begin{gathered}
a_{e e}(u)=\mid\left(x_{w}\left(u_{d}\right)-x(u)\right) \sin \theta(u) \\
-\left(y_{w}\left(u_{d}\right)-y(u)\right) \cos \theta(u) \mid,
\end{gathered}
$$

where $\phi_{\text {st }}(t)$ and $\phi_{\text {ex }}(t)$ represent the instantaneous entrance and exit angles, respectively. $a_{e e}(u)$ represents the instantaneous actual radial depth of cut, which corresponds to $\mathrm{DH}$ in Figure 3. $x_{w}\left(u_{d}\right)$ and $y_{w}\left(u_{d}\right)$ represent the coordinates of Point $D$.

Based on [16], the instantaneous milling force coefficients can be expressed as

$$
\begin{aligned}
& K_{t}\left(h_{i, j}{ }^{\text {total }}(t)\right)=T_{0} h_{i, j}{ }^{\text {total }}(t)^{T_{1}} \\
& K_{r}\left(h_{i, j}{ }^{\text {total }}(t)\right)=R_{0} h_{i, j}{ }^{\text {total }}(t)^{R_{1}},
\end{aligned}
$$

where $T_{0}, T_{1}, R_{0}$, and $R_{1}$ are constants.

Substituting (5) and (13) into (4), milling force of the element $(i, j)$ can be expressed as

$$
\begin{aligned}
& d F_{t i, j}\left(\phi_{s}\left(u_{t}\right)\right)=T_{0}\left(h_{i, j}{ }^{N}(t)+h_{i, j}{ }^{S}(t)\right)^{T_{1}+1} d z \\
& d F_{r i, j}\left(\phi_{s}\left(u_{t}\right)\right)=R_{0}\left(h_{i, j}{ }^{N}(t)+h_{i, j}{ }^{S}(t)\right)^{R_{1}+1} d z .
\end{aligned}
$$

Then, linearizing the milling force by applying Taylor expansion to the infinitesimal elements while neglecting the high order terms and the static part yields

$$
\begin{aligned}
& d F_{t i, j}\left(\varphi_{s}\left(u_{t}\right)\right)=K_{t}^{\prime} h_{i, j}{ }^{S}(t) d z \\
& d F_{r i, j}\left(\varphi_{s}\left(u_{t}\right)\right)=K_{r}^{\prime} h_{i, j}{ }^{S}(t) d z,
\end{aligned}
$$

where $K_{t}^{\prime}=T_{0}\left(T_{1}+1\right)\left[f_{z} \sin \left(\varphi_{s i, j}(t)\right)\right]^{T_{1}}$ and $K_{r}^{\prime}=R_{0}\left(R_{1}+\right.$ 1) $\left[f_{z} \sin \left(\varphi_{s i, j}(t)\right)\right]^{R_{1}}$.
By expressing in the fixed coordinate system (xoy) the cutting force associated with the infinitesimal elements and conducting summation over all the elements, one can obtain the dynamic milling force:

$$
\begin{aligned}
\mathbf{F}= & {\left[\begin{array}{l}
F_{x}(t) \\
F_{y}(t)
\end{array}\right]=\frac{1}{2} d z \mathbf{A}[\mathbf{p}(t)-\mathbf{p}(t-T)] } \\
\mathbf{A}= & {\left[\begin{array}{ll}
a_{x x} & a_{x y} \\
a_{y x} & a_{y y}
\end{array}\right] } \\
a_{x x}= & \sum_{i=1}^{N_{f}} \sum_{j=1}^{M}\left[-K_{t}^{\prime} \sin \left(2 \varphi_{i, j}(t)\right)\right. \\
- & \left.K_{r}^{\prime}\left(1-\cos \left(2 \varphi_{i, j}(t)\right)\right)\right] \\
a_{x y}= & \sum_{i=1}^{N_{f}} \sum_{j=1}^{M}\left[-K_{t}^{\prime}\left(1+\cos \left(2 \varphi_{i, j}(t)\right)\right)\right. \\
- & \left.K_{r}^{\prime} \sin \left(2 \varphi_{i, j}(t)\right)\right] \\
- & \left.K_{r}^{\prime}\left(1+\cos \left(2 \varphi_{i, j}(t)\right)\right)\right], \\
a_{y x}= & \sum_{i=1}^{M} \sum_{j=1}^{M}\left[K_{t}^{\prime}\left(1-\cos \left(2 \varphi_{i, j}(t)\right)\right)\right. \\
- & \left.K_{r}^{\prime} \sin \left(2 \varphi_{i, j}(t)\right)\right] \\
a_{y y}= & \sum_{i=1}^{N_{f}} \sum_{j=1}^{M}\left[K_{t}^{\prime} \sin \left(2 \varphi_{i, j}(t)\right)\right.
\end{aligned}
$$

where $\mathbf{A}$ represents the direction coefficient matrix. $a_{x x}$, $a_{x y}, a_{y x}$, and $a_{y y}$ represent the direction coefficients which are related to the instantaneous tool position and are timedependent while milling a surface with varying curvature.

\section{Analysis of Stability Based on the Time-Domain Semidiscretization Method}

The time-domain semidiscretization method is based on discretizing the delay terms and converting the delay differential equation into a first-order differential equation in the state space, which is in turn solved [4]. Fully accounting for the cutting and noncutting stages of the entire milling process, this method can be widely applied to those cases with various radial depths of cut. Also, it can greatly facilitate the milling stability analysis with varying parameters.

Substituting the dynamic milling force derived from (15) into (3), the delay differential equation of the milling system can be described as

$$
\mathbf{M} \ddot{\mathbf{p}}+\mathbf{C} \dot{\mathbf{p}}+\mathbf{K} \mathbf{p}=\frac{1}{2} d z \mathbf{A}[\mathbf{p}(t)-\mathbf{p}(t-T)] .
$$

By assuming $\mathbf{q}=\left[\begin{array}{l}\mathbf{p} \\ \dot{\mathbf{p}}\end{array}\right]$, the state equation is expressed as

$$
\dot{\mathbf{q}}=\mathbf{L q}(t)+\mathbf{R q}(t-T),
$$


where $\mathbf{L}=\left[\begin{array}{cc}\mathbf{0} & \mathbf{I} \\ (1 / 2) d z \mathbf{M}^{-1} \mathbf{A}-\mathbf{\Omega} & -\boldsymbol{\xi}\end{array}\right]$ represents the system state matrix. $\mathbf{R}=\left[\begin{array}{cc}\mathbf{0} & \mathbf{0} \\ (1 / 2) d z \mathbf{M}^{-1} \mathbf{A} & \mathbf{0}\end{array}\right]$ represents the system input matrix. $\boldsymbol{\Omega}=\left[\begin{array}{cc}k_{x} / m_{x} & 0 \\ 0 & k_{y} / m_{y}\end{array}\right] ; \boldsymbol{\xi}=\left[\begin{array}{cc}c_{x} / m_{x} & 0 \\ 0 & c_{y} / m_{y}\end{array}\right]$; and $\mathbf{M}^{-1}=\left[\begin{array}{cc}1 / m_{x} & 0 \\ 0 & 1 / m_{y}\end{array}\right]$.

The direction coefficient matrix $\mathbf{A}$ is time-dependent for the variable-curvature peripheral milling process; thus, the system is a linear time-dependent system. The delay period $T$ is discretized into $m$ small time elements $(\tau=T / m)$. As for the sampling time $\left(t_{i}=i \tau\right), \mathbf{q}_{i}, \mathbf{L}_{i}$, and $\mathbf{R}_{i}$ represent the corresponding system state, as well as the state and input matrices, respectively; and $\mathbf{q}_{i-m}$ represents $\mathbf{q}\left(t_{i}-T\right)$. If $\tau$ is small enough, $\mathbf{q}\left(t_{i}-T\right) \approx(1 / 2)\left(\mathbf{q}_{i-m}+\mathbf{q}_{i-m+1}\right)$, and (18) is expressed as

$$
\dot{\mathbf{q}}_{i}=\mathbf{L}_{i} \mathbf{q}_{i}+\frac{1}{2} \mathbf{R}_{i}\left(\mathbf{q}_{i-m}+\mathbf{q}_{i-m+1}\right) \quad i \tau \leq t \leq(i+1) \tau .
$$

The solution to (19) is as follows:

$$
\mathbf{q}_{i}(t)=e^{\mathbf{L}_{i}\left(t-t_{i}\right)} \mathbf{C}_{0}-\frac{1}{2} \mathbf{L}_{i}^{-1} \mathbf{R}_{i}\left(\mathbf{q}_{i-m}+\mathbf{q}_{i-m+1}\right),
$$

where $\mathbf{C}_{0}$ is constant and depends on the initial condition of vibration. For $t=i \tau$, the following equation is obtained:

$$
\mathbf{q}_{i}=\mathbf{C}_{0}-\frac{1}{2} \mathbf{L}_{i}^{-1} \mathbf{R}_{i}\left(\mathbf{q}_{i-m}+\mathbf{q}_{i-m+1}\right) \text {. }
$$

Equation (21) can be rewritten as

$$
\mathbf{C}_{0}=\mathbf{q}_{i}+\frac{1}{2} \mathbf{L}_{i}^{-1} \mathbf{R}_{i}\left(\mathbf{q}_{i-m}+\mathbf{q}_{i-m+1}\right) .
$$

For $t=(i+1) \tau$, the following equation is obtained:

$$
\begin{aligned}
\mathbf{q}_{i+1} & =e^{\mathbf{L}_{i} \tau} \mathbf{C}_{0}-\frac{1}{2} \mathbf{L}_{i}^{-1} \mathbf{R}_{i}\left(\mathbf{q}_{i-m}+\mathbf{q}_{i-m+1}\right) \\
& =e^{\mathbf{L}_{i} \tau} \mathbf{q}_{i}+\frac{1}{2}\left(e^{\mathbf{L}_{i} \tau}-\mathbf{I}\right) \mathbf{L}_{i}{ }^{-1} \mathbf{R}_{i}\left(\mathbf{q}_{i-m}+\mathbf{q}_{i-m+1}\right) .
\end{aligned}
$$

Equation (23) can be expressed in a matrix form as

$$
\mathbf{Q}_{i+1}=\Phi_{i} \mathbf{Q}_{i}
$$

where

$$
\begin{aligned}
& \boldsymbol{\Phi}_{i} \\
& =\left[\begin{array}{ccccc}
e^{\mathbf{L}_{i} \tau} & \mathbf{0} & \cdots & \frac{1}{2}\left(e^{\mathbf{L}_{i} \tau}-\mathbf{I}\right) \mathbf{L}_{i}{ }^{-1} \mathbf{R}_{i} & \frac{1}{2}\left(e^{\mathbf{L}_{i} \tau}-\mathbf{I}\right) \mathbf{L}_{i}{ }^{-1} \mathbf{R}_{i} \\
\mathbf{I} & \mathbf{0} & \cdots & \mathbf{0} & \mathbf{0} \\
0 & \mathbf{I} & \cdots & \mathbf{0} & \mathbf{0} \\
\vdots & \vdots & \vdots & \vdots & \vdots \\
0 & 0 & \cdots & \mathbf{I} & \mathbf{0} \\
\mathbf{Q}_{i} & =\left[\begin{array}{c}
\mathbf{q}_{i} \\
\mathbf{q}_{i-1} \\
\vdots \\
\mathbf{q}_{i-m+1} \\
\mathbf{q}_{i-m}
\end{array}\right] .
\end{array}\right.
\end{aligned}
$$

In accordance with the recursive principle, the transfer matrix $(\Phi)$ of the system within a single period $(T)$ can be constructed from $\Phi_{i}(i=1,2, \ldots, m)$ :

$$
\begin{aligned}
\mathbf{Q}_{i+m} & =\Phi Q_{i} \\
\Phi & =\Phi_{i+m-1} \cdots \Phi_{i+1} \Phi_{i} .
\end{aligned}
$$

According to Floquet's theory, the system's stability state depends on the modulus of the maximum eigenvalue of $\Phi$; that is,

$$
\begin{array}{ll}
\max \{|\lambda(\Phi)|\}<1 & \text { stable } \\
\max \{|\lambda(\Phi)|\}=1 & \text { critical } \\
\max \{|\lambda(\Phi)|\}>1 & \text { unstable. }
\end{array}
$$

\section{Location-Dependent Prediction of Stability Limit and Experimental Validation}

Milling system parameters are as follows:

$$
\begin{aligned}
& \text { Milling machine: DMC 70V Hi-Dyn } \\
& \text { Toolholder: HSKA63-ER25-100 } \\
& \text { Cutter: Sandvick 1P240-1000-XA1630 }
\end{aligned}
$$

Diameter: $10 \mathrm{~mm}$

Number of teeth: 4

Helix angle: $35^{\circ}$

Cutter's exposed length postinstallation: $45 \mathrm{~cm}$

With hammering method as shown in Figure 4, the experimental results with DH5920 are shown in Figure 5. The modal parameters of the present tool system can be obtained as follows:

$$
\begin{aligned}
m_{x} & =0.7532, \\
k_{x} & =1.255 \times 10^{7} \mathrm{~N} / \mathrm{m}, \\
c_{x} & =0.036 \\
m_{y} & =0.7689, \\
k_{y} & =1.318 \times 10^{7} \mathrm{~N} / \mathrm{m}, \\
c_{y} & =0.038 .
\end{aligned}
$$

Workpiece material is Al6061-T6.

Experimental identification of the milling force coefficient is linear milling.

Testing parameters are as follows:

Spindle speed: $3000 \mathrm{r} / \mathrm{min}$

Axial depth of cut: $5 \mathrm{~mm}$

Nominal radial depth of cut: $3 \mathrm{~mm}$

Feed per tooth: $0.04 \mathrm{~mm} / \mathrm{r}$

Milling mode is dry cutting and down milling. 


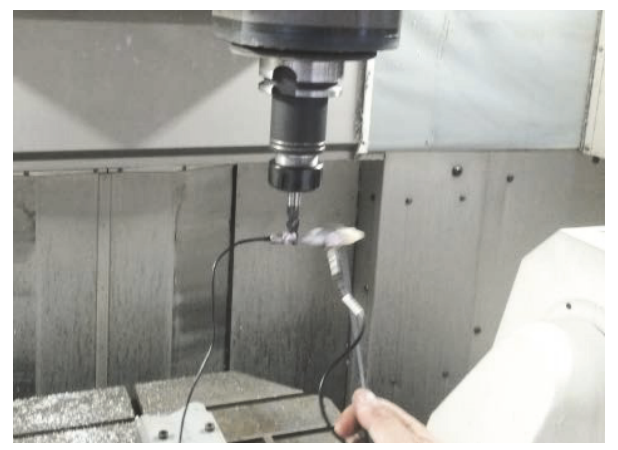

FIGURE 4: Test of modal parameters of tool system.

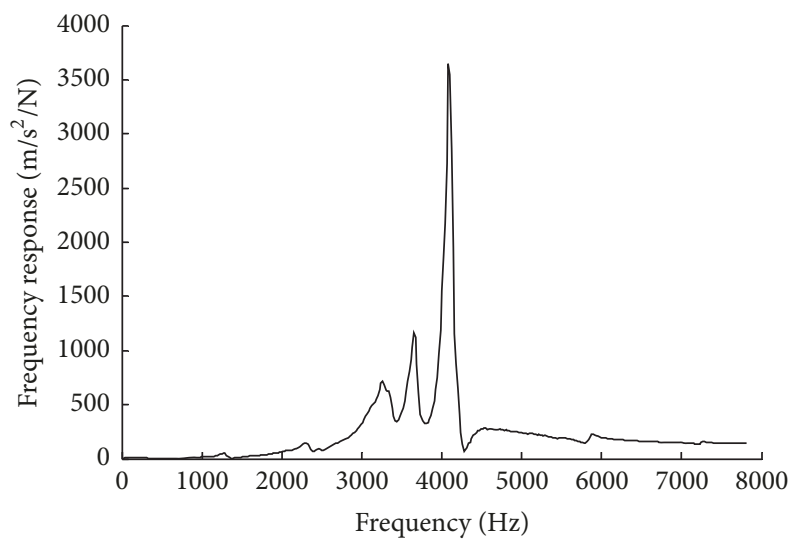

(a) Frequency response functions in $x$ direction

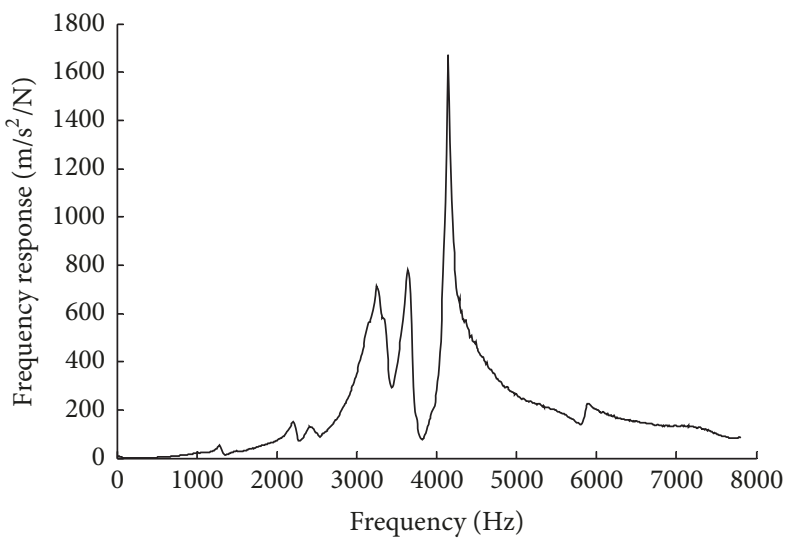

(c) Frequency response functions in $y$ direction

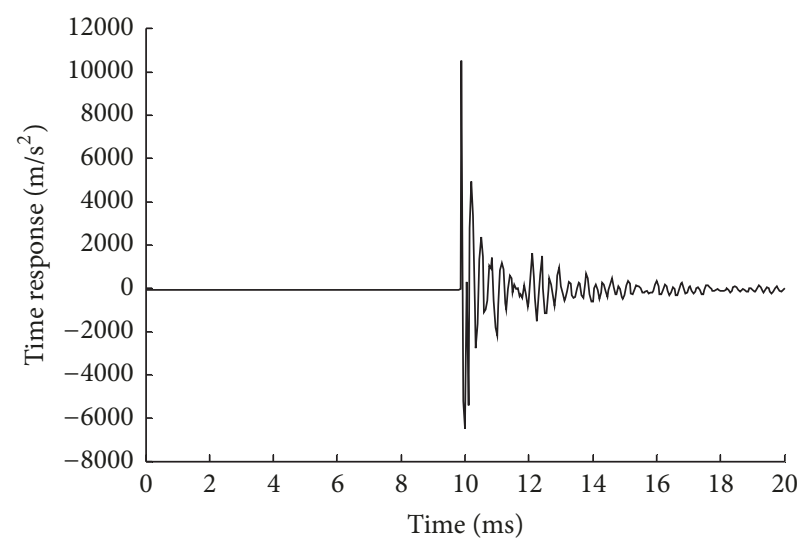

(b) Time response curve in $x$ direction

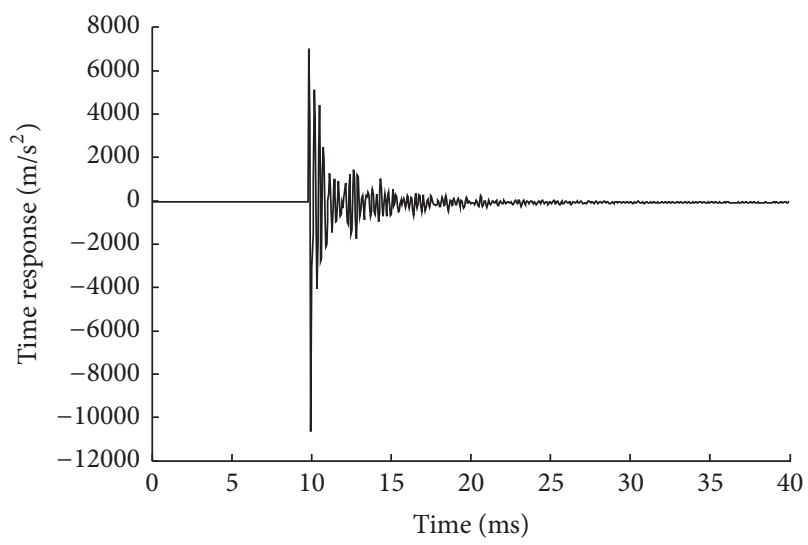

(d) Time response curve in $y$ direction

FIGURE 5: Results of modal experiments with hammering method.

With the method mentioned in [17], milling force coefficients can thereby be calculated as follows:

$$
\begin{aligned}
& k_{t}(h)=432.23 h^{-0.3924} \\
& k_{r}(h)=183.97 h^{-0.469} .
\end{aligned}
$$

4.1. Stability Limit Diagrams. During a variable-curvature peripheral milling process, the feed direction and the actual radial depth of cut are subject to a constant change due to the variation of surface curvature, which in turn causes the associated stability limit to vary accordingly. For a given set of nominal milling parameters, it is possible to encounter a situation where the system is stable during certain periods 
of milling while experiencing vibration during some other periods of milling. Suppose that the workpiece's contour can be expressed using the parametric equation below:

$$
\begin{aligned}
& x(u)=75 u+15 u^{2}-10 u^{3} \\
& y(u)=-120 u+360 u^{2}-240 u^{3}
\end{aligned}
$$

$$
u \in[0,1] .
$$

The milling parameters are set as $f_{z}=0.025 \mathrm{~mm} / \mathrm{r}$ and $a_{e}=2 \mathrm{~mm}$. Based on point-wise scanning method [12], one can compute the stability limits associated with the cutter locations of $(u=0.1,0.3,0.7$, and 0.9$)$; the corresponding feed direction angles and the actual radial depths of cut are listed below:

$$
\begin{aligned}
\theta(0.1) & =-35.4^{\circ}, \\
a_{e e}(0.1) & =2.684 \mathrm{~mm} ; \\
\theta(0.3) & =21^{\circ}, \\
a_{e e}(0.3) & =2.283 \mathrm{~mm} ; \\
\theta(0.7) & =21^{\circ}, \\
a_{e e}(0.7) & =1.737 \mathrm{~mm} ; \\
\theta(0.9) & =-35.4^{\circ}, \\
a_{e e}(0.9) & =1.687 \mathrm{~mm} .
\end{aligned}
$$

The results are shown in Figure 6. A comparison of results between $u=0.1$ and $u=0.9$ indicates that, for the same feed direction angle, the surface curvature affects the effective radial depth of cut, which in turn affects the system's stability limit. Moreover, a comparison of results between $u=0.7$ and $u=0.9$ shows that their stability limit diagrams differ dramatically despite the fact that their effective radial depths of cut are quite close, which indicates that the feed direction also affects the stability to some extent.

For a given set of spindle speed, nominal radial depth of cut, and feed per tooth, one can place the cutting tool at different locations and use (27) to calculate the corresponding maximum radial depths of cut based on instantaneous effective radial depth of cut and feed direction, with the results shown in Figure $7\left(f_{z}=0.025 \mathrm{~mm} / \mathrm{r}, a_{e}=2 \mathrm{~mm}\right.$, and $n_{r}=$ $4800 \mathrm{r} / \mathrm{min}$ ). It can be seen in the figure that, for the given milling parameters, the axial depths of cut must be less than $2.8 \mathrm{~mm}$ to ensure the stability during the entire milling process.

Similarly, the maximum nominal radial depths of cut corresponding to various cutter locations are calculated for a given set of spindle speed, axial depth of cut, and feed per tooth, with the results shown in Figure $8\left(f_{z}=0.025 \mathrm{~mm} / \mathrm{r}\right.$, $a_{p}=2 \mathrm{~mm}$, and $\left.n_{r}=4800 \mathrm{r} / \mathrm{min}\right)$. It can be seen in the figure that the maximum nominal radial depth of cut must be less than $3.6 \mathrm{~mm}$ to ensure the stability during the entire milling process.

For a given combination of spindle speed, axial depth of cut, and nominal radial depth of cut, the maximum feeds

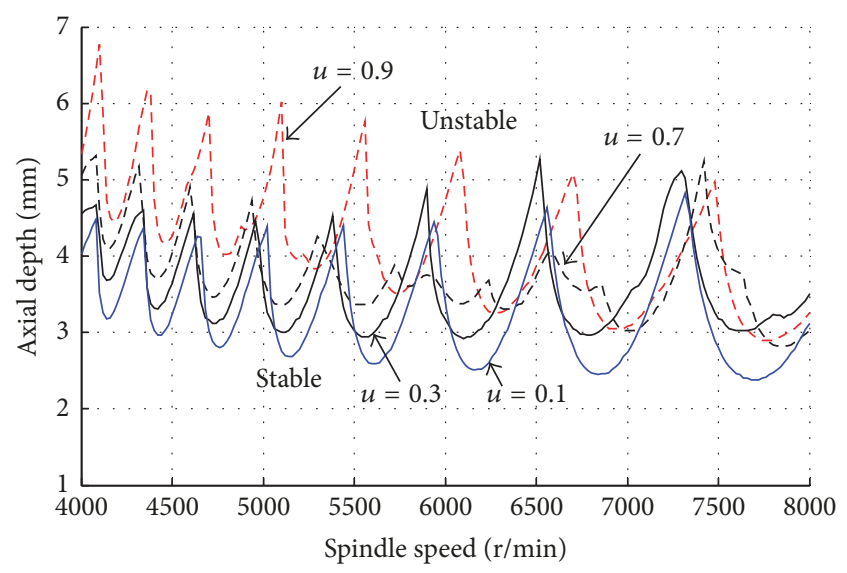

FIGURE 6: Stability limit diagram when the tool cutting tool position.

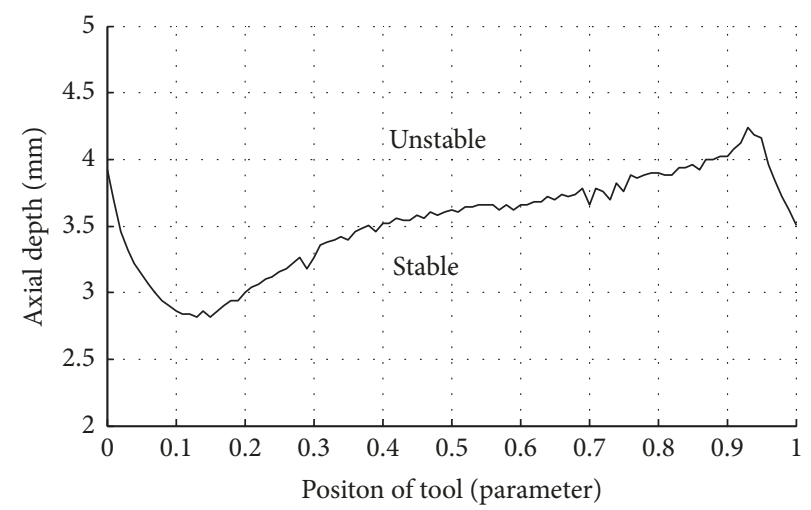

Figure 7: Maximum axial cutting depth versus tool position.

per tooth corresponding to various cutter positions are calculated, with the results shown in Figure $9\left(n_{r}=4800 \mathrm{r} / \mathrm{min}\right.$, $a_{p}=3 \mathrm{~mm}$, and $a_{e}=2 \mathrm{~mm}$ ). It can be seen in the figure that the feed per tooth must be more than $0.028 \mathrm{~mm} / \mathrm{r}$ to ensure the stability during the entire milling process.

4.2. Experimental Validation. For verifying the correctness of location-dependent prediction of the variable-curvature milling stability, a test is designed in accordance with the predicted results (Figure 7). The experimental scene is shown in Figure 10. The test parameters are $f_{z}=0.025 \mathrm{~mm} / \mathrm{r}, a_{e}=$ $2 \mathrm{~mm}$, and $n_{r}=4800 \mathrm{r} / \mathrm{min}$, and the axial depths of cut are $a_{p}=2.7 \mathrm{~mm}$ and $a_{p}=2.9 \mathrm{~mm}$, respectively. The maximum axial cutting depth reaches its lowest level for a cutter position of $u=0.13$ and the corresponding machining duration is $1.95 \mathrm{~s}$. Thus, analysis is carried out concerning the signals of $y$-direction milling force near $t=1.95 \mathrm{~s}$ and the results are shown in Figure 11. As we know, when milling is stable, frequency arising in frequency spectrum of cutting force signal is cutting frequency or it is harmonic frequency. According to the milling parameters, cutting frequency is $320 \mathrm{~Hz}$. It can be seen in Figure 11 that the milling stability is achieved at those cutting frequencies marked in the frequency spectrum of milling force (i.e., $320 \mathrm{~Hz}$ and its multiplications) when the axial depth of cut is $2.7 \mathrm{~mm}$. When the axial depth of cut is 


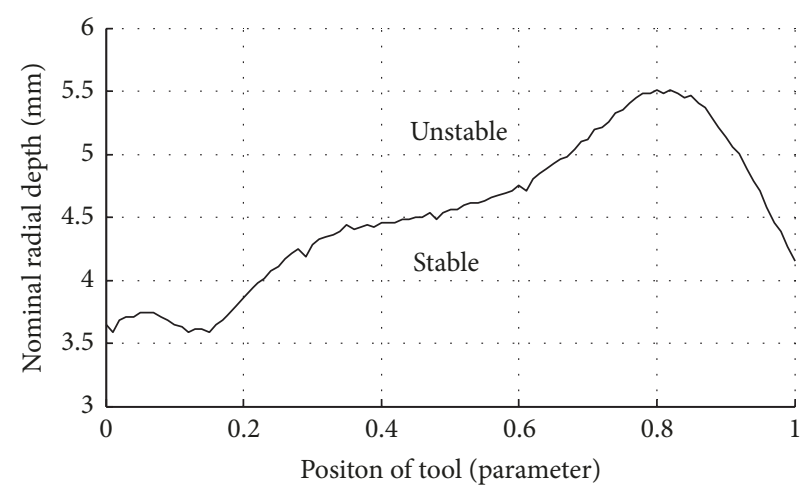

FIgURE 8: Maximum nominal radial cutting depth versus tool position.

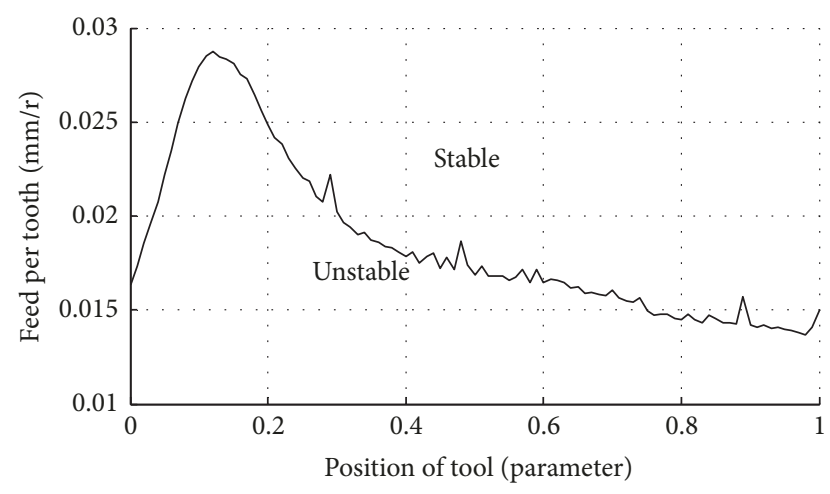

FIGURE 9: Maximum feed per tooth versus tool position.

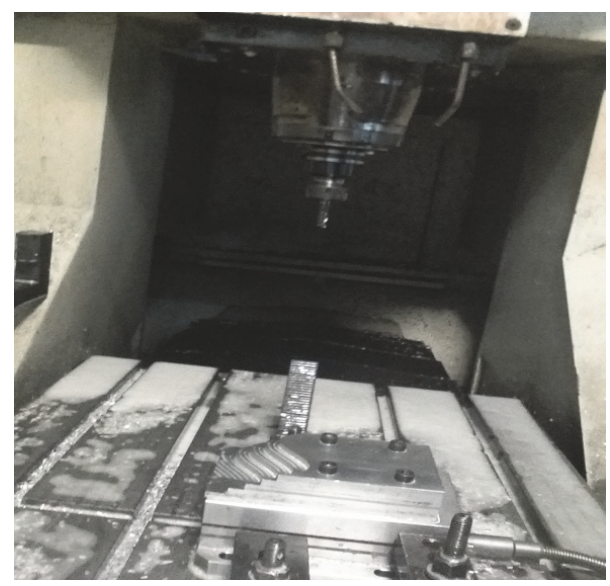

FIgUre 10: Milling tests.

$2.9 \mathrm{~mm}$, it is obvious that the cutting vibration frequencies are $4147 \mathrm{~Hz}, 4707 \mathrm{~Hz}$, and $5027 \mathrm{~Hz}$. Their spacing is the integer multiples of the cutter's rotating frequency. This indicates that an unstable stage is encountered during the milling process under the given machining conditions. The measured results at two axial depths of cut indicate that our method for conducting location-dependent prediction concerning the stability of peripheral milling over surfaces with variable curvatures is accurate and feasible.

It should be noted that the cutter runout has a certain effect on milling stability, because runout makes the uncut chip thickness deviate from the nominal value. Wan has analyzed the effect of runout on milling stability in [18]. In this paper, the main purpose is to provide a method for locationdependent prediction of dynamic stability. To simplify the dynamic model solution, the runout is neglected.

\section{Conclusions}

By considering the impact of surface curvature on the effective radial depth of cut, the present study proposes a method for computing the effective radial depth of cut, which can be employed to rapidly determine the entrance/exit angles based on the effective radial depth of cut. As a result, the computational overhead for predicting the milling force is greatly reduced, and the computational efficiency is substantially enhanced; moreover, a dynamic milling model is established based on the instantaneous milling force coefficients, which is solved using the time-domain semidiscretization method to determine the milling stability; in addition, a method for conducting location-dependent prediction of stability related to the peripheral milling of complex surfaces is put forward by fully taking into consideration the complex surface milling stability's dependency on cutter location. Furthermore, simulation concerning the peripheral milling of complex surfaces is carried out, based on which the stability limit diagrams pertaining to axial and radial depths of cut as well as the feed per tooth are plotted. Also, the effectiveness of the proposed method is validated with the milling experiments.

\section{Data Availability}

The data used to support the findings of this study are available from the corresponding author upon request. 


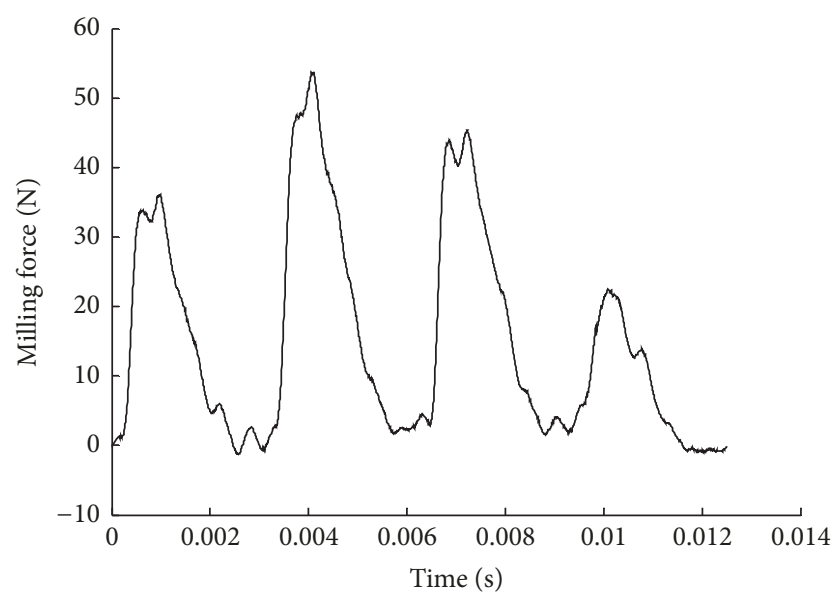

(a) Milling force in $y$-direction $\left(a_{p}=2.7 \mathrm{~mm}\right)$

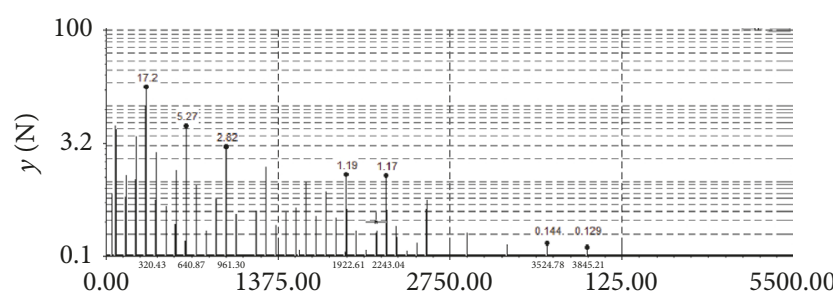

(c) Frequency spectrum of $y$-direction milling force $\left(a_{p}=2.7 \mathrm{~mm}\right)$

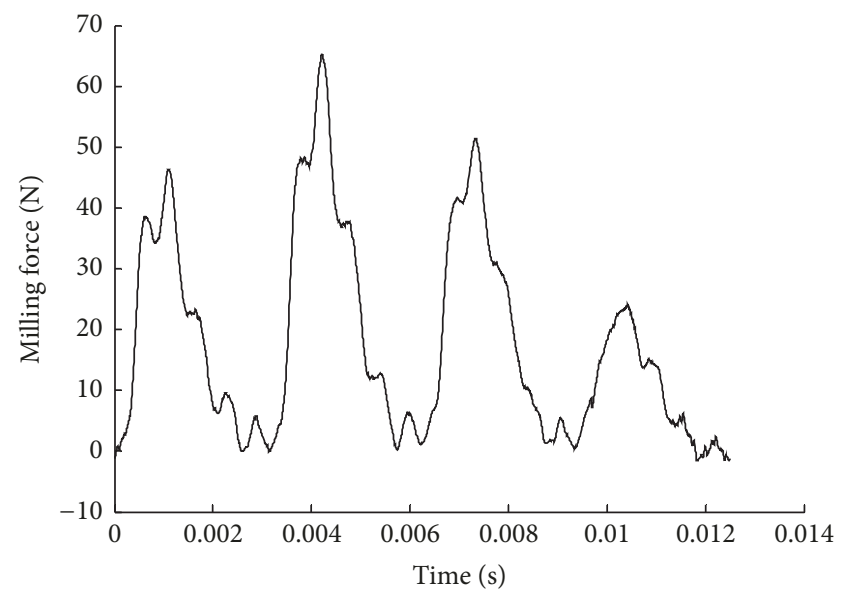

(b) Milling force in $y$-direction $\left(a_{p}=2.9 \mathrm{~mm}\right)$

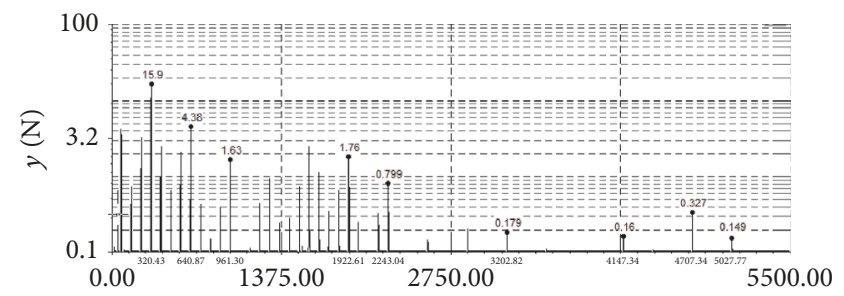

(d) Frequency spectrum of $y$-direction milling force $\left(a_{p}=2.9 \mathrm{~mm}\right)$

FIGURE 11: Frequency spectrum of $y$-direction milling force.

\section{Conflicts of Interest}

The authors declare that there are no conflicts of interest regarding the publication of this article.

\section{Acknowledgments}

This work was financially supported by the Joint Research Project of Jiangsu Province, China (BY2016008-04), the University Science Research Project of Jiangsu Province, China (16KJD460003), and the Scientific Foundation of Nanjing Institute of Technology (ZKJ201201).

\section{References}

[1] Y. Altintaş and E. Budak, "Analytical prediction of stability lobes in milling," CIRP Annals - Manufacturing Technology, vol. 44, no. 1, pp. 357-362, 1995.

[2] E. Budak and Y. Altıntaş, "Analytical prediction of chatter stability in milling - part I: general formulation," Journal of Dynamic Systems, Measurement and Control-Transactions of the ASME, vol. 120, no. 1, pp. 22-30, 1998.

[3] S. D. Merdol and Y. Altintas, "Multi frequency solution of chatter stability for low immersion milling," Journal of Manufacturing Science and Engineering, vol. 126, no. 3, pp. 459-466, 2004.

[4] T. Insperger and G. Stepan, "Semi-discretization method for delayed systems," International Journal for Numerical Methods in Engineering, vol. 55, no. 5, pp. 503-518, 2002.
[5] M. Zatarain, J. Muñoa, G. Peigne, and T. Insperger, "Analysis of the influence of mill helix angle on chatter stability," CIRP Annals - Manufacturing Technology, vol. 55, no. 1, pp. 365-368, 2006.

[6] X. Long and B. Balachandran, "Stability of up-milling and downmilling operations with variable spindle speed," Journal of Vibration and Control, vol. 16, no. 7-8, pp. 1151-1168, 2010.

[7] Q. Guo, Y. Sun, Y. Jiang, and D. Guo, "Prediction of stability limit for multi-regenerative chatter in high performance milling," International Journal of Dynamics and Control, vol. 2, no. 1, pp. 35-45, 2014.

[8] Y. Ding, L. Zhu, X. Zhang, and H. Ding, "A full-discretization method for prediction of milling stability," The International Journal of Machine Tools and Manufacture, vol. 50, no. 5, pp. 502-509, 2010.

[9] C. G. Ozoegwu, S. N. Omenyi, and S. M. Ofochebe, "Hyper-third order full-discretization methods in milling stability prediction," The International Journal of Machine Tools and Manufacture, vol. 92, pp. 1-9, 2015.

[10] M. Zatarain, J. Alvarez, I. Bediaga, J. Munoa, and Z. Dombovari, "Implicit subspace iteration as an efficient method to compute milling stability lobe diagrams," The International Journal of Advanced Manufacturing Technology, vol. 77, no. 1-4, pp. 597607, 2015.

[11] R. G. Landers and A. G. Ulsoy, "Nonlinear feed effect in machining chatter analysis," Journal of Manufacturing Science and Engineering, vol. 130, pp. 1707-1708, 2008.

[12] K. Ahmadi and F. Ismail, "Modeling chatter in peripheral milling using the Semi Discretization Method," CIRP Journal of 
Manufacturing Science and Technology, vol. 5, no. 2, pp. 77-86, 2012.

[13] N. Kardes and Y. Altintas, "Mechanics and dynamics of the circular milling process," Journal of Manufacturing Science and Engineering, vol. 129, no. 1, pp. 21-31, 2007.

[14] C. Peng, L. Wang, Z. Li, and Y. Yang, "Time-domain simulation and experimental verification of dynamic cutting forces and chatter stability for circular corner milling," Proceedings of the Institution of Mechanical Engineers, Part B: Journal of Engineering Manufacture, vol. 229, no. 6, pp. 932-939, 2015.

[15] Y. Yang, W.-H. Zhang, Y.-C. Ma, and M. Wan, "Chatter prediction for the peripheral milling of thin-walled workpieces with curved surfaces," The International Journal of Machine Tools and Manufacture, vol. 109, pp. 36-48, 2016.

[16] B. Wang, H. Hao, M. Wang, J. Hou, and Y. Feng, "Identification of instantaneous cutting force coefficients using surface error," The International Journal of Advanced Manufacturing Technology, vol. 68, no. 1-4, pp. 701-709, 2013.

[17] H. Hao, B. Wang, and W. Tang, "Prediction of instantaneous milling force taking runout into account in peripheral milling of curved surface," The International Journal of Advanced Manufacturing Technology, vol. 79, no. 1-4, pp. 49-56, 2015.

[18] M. Wan, W. H. Zhang, J. W. Dang, and Y. Yang, "A unified stability prediction method for milling process with multiple delays," in Proceedings of the International Journal of Machine Tools Manufacture 50, vol. 50, pp. 29-41, 2010. 


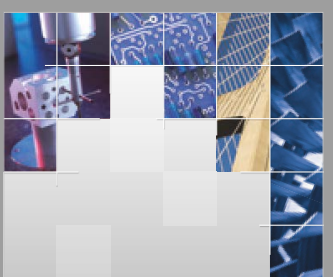

\section{Enfincering}
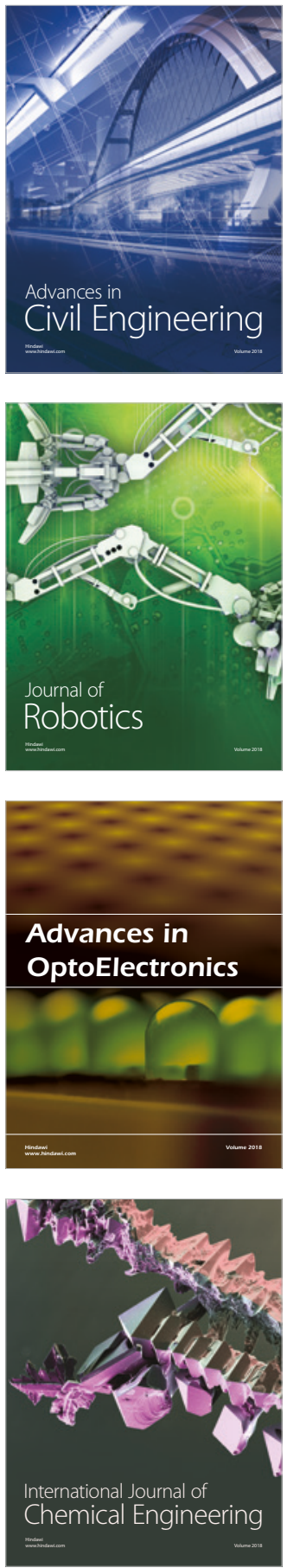

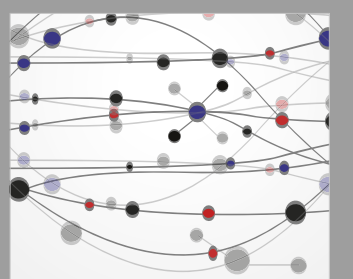

\section{Rotating \\ Machinery}

The Scientific World Journal

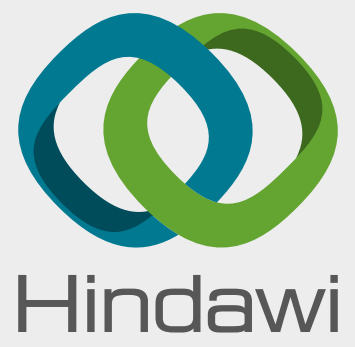

Submit your manuscripts at

www.hindawi.com
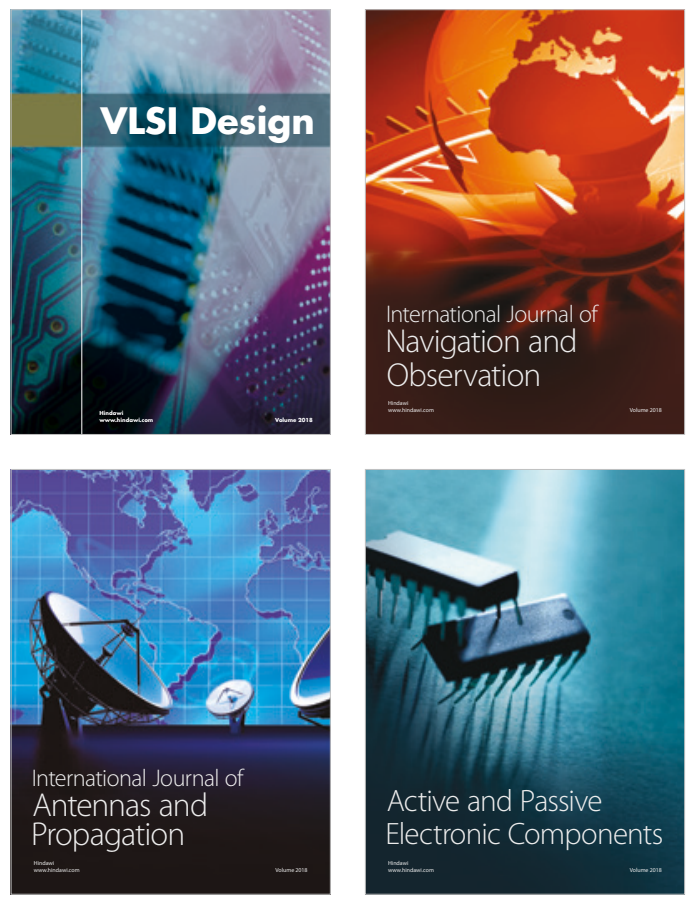
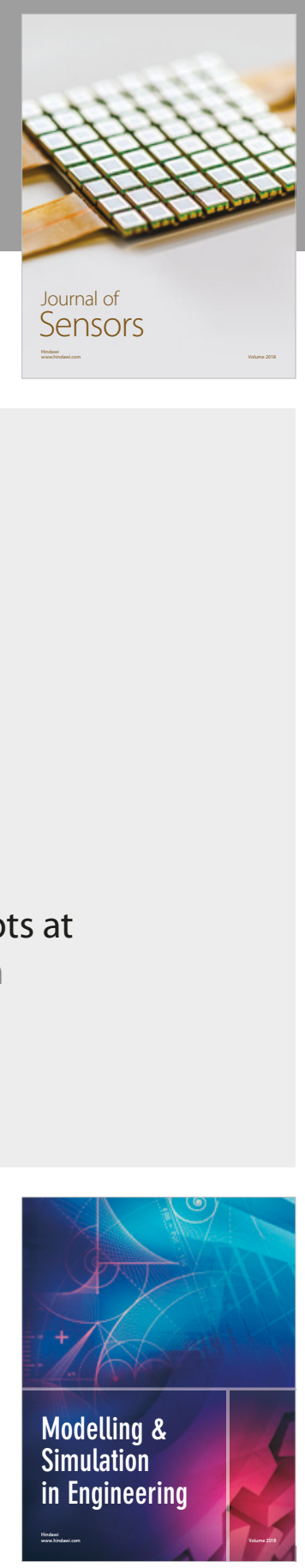

\section{Advances \\ Multimedia}
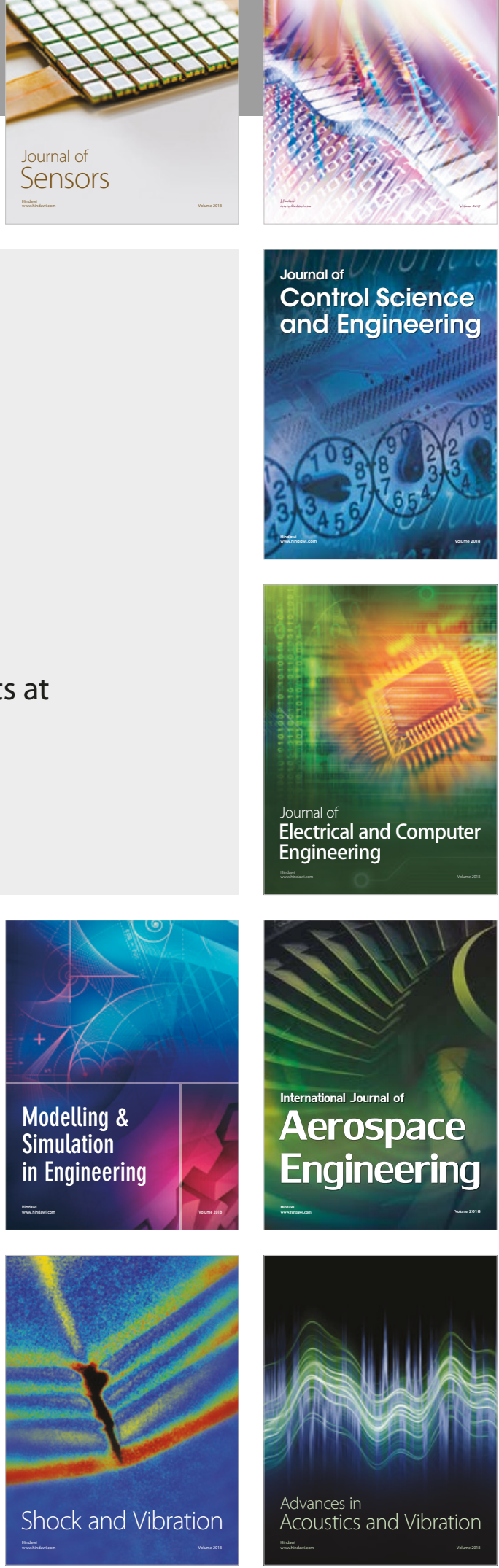